\title{
Preparation and properties of biodegradable starch-clay nanocomposites
}

\author{
Yi-Lin Chung ${ }^{a}$, Seema Ansari ${ }^{\mathrm{b}}$, Luis Estevez ${ }^{\mathrm{b}}$, Suren Hayrapetyan ${ }^{\mathrm{b}}$, Emmanuel P. Giannelis ${ }^{\mathrm{b}, *}$, Hsi-Mei Lai ${ }^{\mathrm{a}, *}$ \\ ${ }^{a}$ Department of Agricultural Chemistry, National Taiwan University, No. 1, Roosevelt Rd., Sec. 4, Taipei 10617, Taiwan \\ ${ }^{\mathrm{b}}$ Department of Materials Science and Engineering, Cornell University, Ithaca, NY 14853, USA
}

\section{A R T I C L E I N F O}

Article history:

Received 14 July 2009

Received in revised form 14 August 2009

Accepted 17 August 2009

Available online 20 August 2009

\section{Keywords:}

Starch

Clay

Nanocomposites

Biodegradable

Bioplastics

\begin{abstract}
A B S T R A C T
Well-dispersed starch-clay nanocomposites were prepared by adding a dilute clay dispersion to a solution of starch followed by coprecipitation in ethanol. The clay didn't significantly influence the type of crystalline structure of starch molecules although the amount of crystallinity appears to be somewhat lower in the nanocomposites. The nanocomposites show improved modulus and strength without a decrease in elongation at break. The increase in modulus and strength is $65 \%$ and $30 \%$, respectively for the nanocomposite containing $5 \mathrm{wt}$.\% clay compared to the unfilled starch materials. Further increases in clay result in deterioration in properties most likely due to poorer clay dispersion and lower polymer crystallinity. As the amount of water increases, the modulus of both pure starch and starch nanocomposites decreases, although the change is less pronounced in the nanocomposites suggesting that the addition of clay to form nanocomposites can improve the stability of starch-based products during transportation and storage.
\end{abstract}

(c) 2009 Elsevier Ltd. All rights reserved.

\section{Introduction}

Most synthetic polymers are produced from petrochemicals and are harmful to nature. Their synthesis produces hazardous waste and these materials are not easily degradable, causing environmental problems. Plants are potential sources for a wide variety of polymers which are renewable and ecologically friendly. The agro-based biopolymers are edible, biocompatible and biodegradable, which make them superior to synthetic polymers and particularly useful in disposable plastics, food, and medicine applications. Starches, the dominant carbohydrate reserve materials of higher plants, are abundant and relatively inexpensive biopolymers. They contain stored energy from sun and can be produced steadily without fears of exhaustion. Furthermore, in the presence of plasticizers, starches can be processed using conventional thermoplastic techniques. However, the mechanical properties of starch-based materials are often sensitive and responsive to the changes in the environment such as humidity, temperature, and $\mathrm{pH}$. This drawback has to be overcome to obtain high performance biomaterials.

Biopolymer-clay nanocomposites are a new class of materials with potentially improved mechanical properties. These composites are prepared by addition of low amounts of clay to the biopolymer matrix (Zhao, Torley, \& Halley, 2008). The main challenge for

\footnotetext{
* Corresponding authors. Tel.: +1 6072559680 (E.P. Giannelis); tel.: +886 2 33664816; fax: +886 23633123 (H.-M. Lai).

E-mail addresses: epg2@cornell.edu (E.P. Giannelis), hmlai@ntu.edu.tw (H.-M.
}

preparing nanocomposites is the nanoscale dispersion of clay in the biopolymer matrix. Montmorillonite is the most commonly used natural clay and has been successfully applied in numerous nanocomposite systems (Giannelis, 1996; Paul \& Robeson, 2008; Pavlidou \& Papaspyrides, 2008; Raquez, Narayan, \& Dubois, 2008; Ray \& Okamoto, 2003). However, most of the reported starch-clay nanocomposites suffer from poor dispersion, which is required for obtaining high performance materials (Bagdi, Muller, \& Pukanszky, 2006; Chiou et al., 2006; Pandey \& Singh, 2005; Park, Lee, Park, Cho, \& Ha, 2003; Park et al., 2002; Wilhelm, Sierakowski, Souza, \& Wypych, 2003a, 2003b). To improve the dispersion, organic cations such as stearyl dihydroxyethyl ammonium chloride (Bagdi et al., 2006), distearyl dimethyl ammonium chloride (Bagdi et al., 2006) and quaternary ammonium-modified starches (Chivrac et al., 2008) were used to exchange with the sodium ions residing in the interlayer of pristine montmorillonite. The more the modifier is compatible with starch, the more it facilitates clay dispersion (Chivrac et al., 2008). However, miscibility is still an issue and clay dispersion remains a challenge (Bagdi et al., 2006; Chiou et al., 2006; Park et al., 2002, 2003).

Plasticizer is another important factor that influences the properties of starch-clay nanocomposites. The melting temperature of a starch granule is close to its degradation temperature. Hence, plasticizers are required to destroy the inter-molecular hydrogen bonding in the crystalline regions of starch granules and decrease the melting temperature during thermoplastic processing. Small molecules such as glycerol and water that can form hydrogen bonds with starch can serve as plasticizers in starch-based materials. A part of the plasticizers can potentially be absorbed on the 
clay surface, influencing the interfacial strength and stress transfer between the polymeric matrix and clay (Chivrac, Pollet, Schmutz, \& Averous, 2008). Depending on the relative humidity, the moisture content (plasticizer concentration) of the starch-clay nanocomposites will change and consequently influence the Young's modulus of the materials (Avella et al., 2005; Chivrac, Pollet et al., 2008; Huang, Yu, \& Ma, 2006; Ma, Yu, \& Wang, 2007; Perez, Alvarez, Mondragon, \& Vazquez, 2008).

In this study, we prepared well-dispersed starch-clay nanocomposites with three different kinds of clay: montmorillonite, chitosan-modified montmorillonite, and laponite. Chitosan-modified montmorillonite is of interest because chitosan is a natural polysaccharide that is compatible with the starch matrix as well as being ion-exchanged in the clay (Darder, Colilla, \& Ruiz-Hitzky, 2003; Kampeerapappun, Aht-ong, Pentrakoon, \& Srikulkit, 2007). Laponite is a synthetic silicate clay with a smaller aspect ratio (20-30 $\mathrm{nm}$ in the planar dimension and $\sim 1 \mathrm{~nm}$ in the thickness) and purer than montmorillonite. Well-dispersed nanocomposites were produced, and the silicate dispersion from nanometer to micrometer scales was characterized using XRD, TEM, and SEM. Furthermore, the mechanical properties of starch-montmorillonite nanocomposites with various clay concentrations were investigated. In order to understand the interactions of starch matrix with plasticizers and clay, mechanical properties of the nanocomposites were evaluated with respect to relative humidity $(0-75 \% \mathrm{RH})$ which also provides an insight to their mechanical response to the environmental changes.

\section{Experimental}

\subsection{Materials}

Native normal corn starch (NCS, Cargill Gel ${ }^{\mathrm{TM}} 03420$ ) was kindly provided by Cargill Co. (USA). Sodium montmorillonite ( $\mathrm{Na}^{+} \mathrm{MMT}$ ) and laponite RD were kindly supplied by Southern Clay Products, Inc. (USA). Pure glycerol (99.5\%) and chitosan with molecular weight 5-190 kDa (Sigma-Aldrich, USA) were used as received.

\subsection{Sample preparation}

\subsubsection{Preparation of chitosan-modified montmorillonite}

Chitosan solutions were prepared by adding $1 \mathrm{~g}$ of polysaccharide to $1 \%$ acetic acid $(100 \mathrm{~mL})$. The solutions were stirred at $60{ }^{\circ} \mathrm{C}$ for $1 \mathrm{~h}$, followed by continuously stirring overnight at room temperature. The $\mathrm{pH}$ of the solution was adjusted to 4.9 with $\mathrm{NaOH}$. A stock of well-dispersed clay suspension was prepared by adding sodium montmorillonite in water and keeping at $60{ }^{\circ} \mathrm{C}$ overnight with stirring, followed by centrifugation $(117 \mathrm{~g})$ for five minutes to discard any solids. Chitosan solutions were mixed with sodium montmorillonite dispersions $(100 \mathrm{~mL})$ at $60^{\circ} \mathrm{C}$ for 1 day. The mixture was centrifuged at $2935 \mathrm{~g}$ for $5 \mathrm{~min}$. The precipitates were washed with distilled water five times and the supernatants, containing excess chitosan, were discarded. The recovered solids were then dried at $50{ }^{\circ} \mathrm{C}$ in a vacuum oven. The dried solids were ground into powders and passed through a 60 mesh screen. To disperse the chitosan-modified montmorillonite, $1.5 \mathrm{~g}$ of the powder were added to distilled water $(98.5 \mathrm{~mL})$, and the suspension was sonicated for $22 \mathrm{~h}$. The supernatant was collected after sedimentation at room temperature for $30 \mathrm{~min}$.

\subsubsection{Synthesis of starch-clay nanocomposites}

To completely gelatinize starch granules, $2 \mathrm{~g}$ of native normal corn starches were suspended in distilled water $(200 \mathrm{~mL})$ and heated at $100{ }^{\circ} \mathrm{C}$ for $30 \mathrm{~min}$. An aqueous clay dispersion containing $0.02-0.14 \mathrm{~g}$ of clay was slowly added to the starch solution. The mixture was stirred at $90{ }^{\circ} \mathrm{C}$ for $4 \mathrm{~h}$. The clay polymer mixture was precipitated by adding an equal volume of $95 \%$ ethanol and storing at $4{ }^{\circ} \mathrm{C}$ overnight. The precipitate was centrifuged for $5 \mathrm{~min}$ and vacuum dried at $50^{\circ} \mathrm{C}$. The nanocomposites were blended with plasticizers (nanocomposites: water:glycerol = 100:40:30) in a speed mixer and kept at room temperature for 2 days to swell the starch molecules. Films were obtained by hotpressing at $100^{\circ} \mathrm{C}$ using $30 \mathrm{kPa}$ pressure for $3 \mathrm{~min}$ and stored at $53 \% \mathrm{RH}$ (relative humidity).

\subsection{Characterization}

\subsubsection{X-ray diffraction (XRD)}

$X$-ray diffraction analysis was performed on a Scintag ThetaTheta diffractometer (USA) with $\mathrm{CuK}_{\alpha}$ radiation $(\lambda=1.5406 \AA)$ operating at $45 \mathrm{kV}$ and $40 \mathrm{~mA}$. Scans were made in the range of $2 \theta=1.5-40^{\circ}$ by steps of $0.02^{\circ}$ of $0.5 \mathrm{~s}$ each. Slits of $1^{\circ}$ and $3^{\circ}$ for the source and $0.3^{\circ}$ and $0.5^{\circ}$ for the detector, respectively were used.

\subsubsection{Transmission electron microscopy (TEM)}

Samples were microtomed at room temperature using a Leica Ultracut UCT Cryo ultramicrotome (Germany) equipped with a sapphire knife. Ultrathin sections $(\sim 100 \mathrm{~nm})$ were picked up using a copper grid with acetone on the surface. The samples were examined using a FEI Techai G2 T12 Spirit TEM (USA) operating at an acceleration voltage of $120 \mathrm{kV}$.

\subsubsection{Scanning electron microscopy (SEM)}

Fractured surfaces prepared in liquid nitrogen were studied using LEO-1550 Keck Field Emission SEM (Germany) operating at an acceleration voltage of $3 \mathrm{kV}$. The samples were fractured under liquid nitrogen and then dried at $40-60{ }^{\circ} \mathrm{C}$ for 2 days under vacuum. The dried samples were coated with a thin $\mathrm{Au} / \mathrm{Pt}$ film to avoid charging.

\subsubsection{Mechanical testing}

Tensile tests were carried out using a Dynamic Mechanical Analysis (DMA2980, film/fiber tension clamp, TA instruments, USA) operating under a stress/strain mode. The dumbbell-shaped specimens were conditioned at room temperature under 0,43 , or $75 \% \mathrm{RH}$ for 3 days. Then, the samples were equilibrated at $25^{\circ} \mathrm{C}$ for $5 \mathrm{~min}$ prior to testing. A constant deformation force of $3 \mathrm{~N} / \mathrm{s}$ was used. Three different batches of nanocomposites were prepared and six specimens from each batch were tested and analyzed.

\subsubsection{Moisture sorption isotherm}

The samples were dried in a chamber containing drierite (W.A.HAMMOND Co. Ltd., USA) for 5 days before being equilibrated at different RH. Different saturated salt solutions $\left(\mathrm{K}_{2} \mathrm{COOH}\right.$, $\left.\mathrm{K}_{2} \mathrm{CO}_{3}, \mathrm{Mg}\left(\mathrm{NO}_{3}\right)_{2}, \mathrm{NaCl}, \mathrm{H}_{2} \mathrm{O}\right)$ were prepared in the individual sealed containers to create the specific RH $(23,43,53,75$, and $100 \%)$ at $25^{\circ} \mathrm{C}$. The moisture content was calculated from the increase in mass of the dried sample after equilibration at a given $\mathrm{RH}$ condition.

\section{Results and discussion}

\subsection{Synthesis of starch-clay nanocomposites}

There are two basic structures reported for polymer-clay nanocomposites. In intercalated nanocomposites the polymer chains penetrate between the silicate layers keeping the clay stacks (tactoids) virtually unchanged except for an increase in the repeat 
distance ( $d$-spacing). In exfoliated nanocomposites, the clay layers are delaminated and separated from one another by a distance greater than a few nanometers with no memory of the original clay tactoids. It is difficult to obtain either an intercalated or exfoliated structure in starch-clay nanocomposites. This is because corn starch is a neutral polymer which lacks the driving force to replace sodium ions in the interlayer spacing through an ion exchange reaction. Moreover, corn starches are high molecular weight $\left(1 \times 10^{6}\right.$ to $\left.10^{8}\right)$ polymers and are composed of anhydroglucose units which are connected through $\alpha-(1 \rightarrow 4)$ and $\alpha-(1 \rightarrow 6)$-linkages. These rigid structures make it difficult for starches to intercalate into the galleries between the clay layers. Also starches are usually processed with plasticizers during extrusion and hotpressing. Plasticizers and starch molecules can compete to intercalate into the clay (Pandey \& Singh, 2005). The interaction between plasticizers and clays tends to be more favorable because of the smaller molecular size of plasticizers. Thus, the addition of plasticizers such as glycerol might inhibit intercalation and exfoliation of clays in the starch matrix.

To circumvent some of these problems a clay suspension containing a lower percentage of clay $(0.2-1.4 \mathrm{wt} . \%)$ from that typically used was prepared to promote clay dispersion. A low concentration starch solution was also prepared to decrease the viscosity and increase the miscibility with clay. We hypothesized that, during precipitation, the well-dispersed clay layers can be trapped between the starch molecules. The plasticizer (glycerol) was then added into the dispersed starch-clay system to prevent as much as possible intercalation of glycerol into the clay and promote starch-clay interactions. A schematic of the synthesis is shown in Fig. 1.

The X-ray diffraction patterns of clay are shown in Fig. 2. The diffraction peak of montmorillonite at $7.3^{\circ}\left(d_{001}=1.2 \mathrm{~nm}\right)$ corresponds to a clay intercalated with a monolayer of water. The chitosan-modified montmorillonite displays a peak at $3.9^{\circ}$ corresponding to a $d_{001}$ value of $22 \AA$. We attribute the increase of the interlayer spacing to the intercalation of chitosan into the clay (Darder et al., 2003). The laponite sample displays a wide diffraction peak at $6.6^{\circ}$ corresponding to a $d_{001}$ interlayer spacing of $1.4 \mathrm{~nm}$, which corresponds to a clay intercalated with approximately two layers of water molecules.

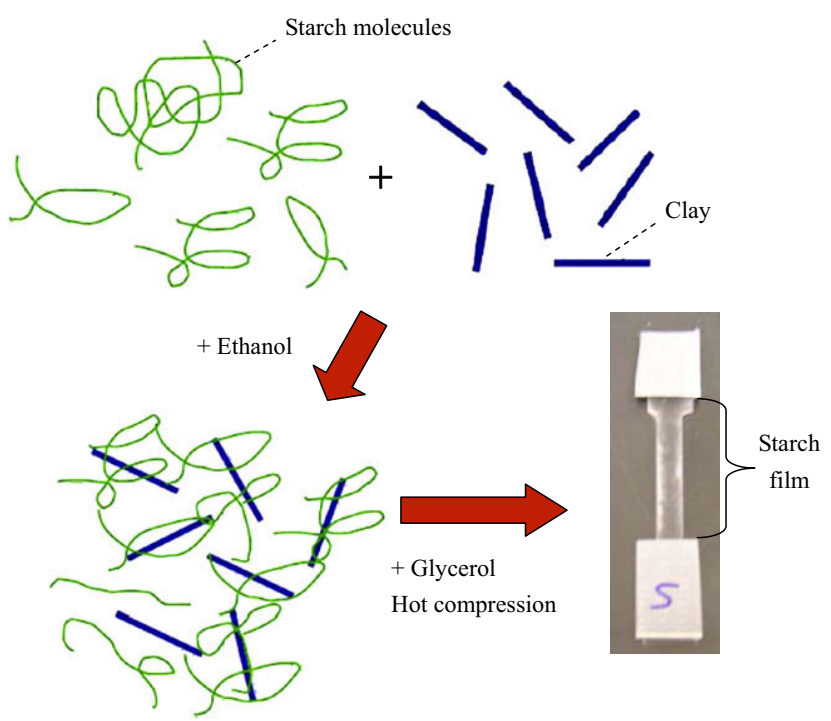

Fig. 1. Schematic representation of the synthesis of well-dispersed starch-clay nanocomposites.

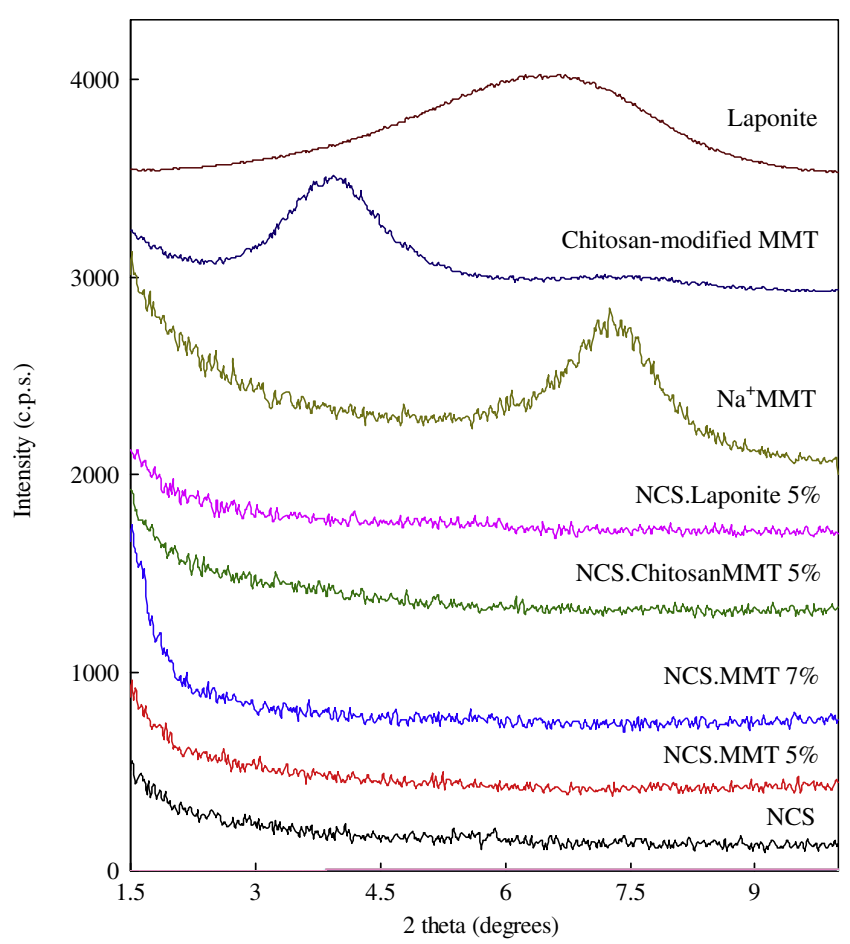

Fig. 2. XRD patterns for montmorillonite, laponite, montmorillonite modified with chitosan, and various starch-clay nanocomposites.

\subsection{Dispersion of silicate layers}

The dispersion of clay in the nanocomposite powders and films was investigated using X-ray diffractometry and electron microscopy. Fig. 2 shows the XRD patterns of starch-clay nanocomposite films. There are no diffraction peaks in the $2 \theta$ range of $1.5^{\circ}-10^{\circ}$ for all powder and film samples. The characteristic peaks corresponding to clay are not observed even for nanocomposites containing 7 wt.\% clay. The loss of the $d_{001}$ peak of the clay is attributed to the good clay dispersion leading to disordered clay tactoids, and low concentration of clay agglomerates. Intercalation of glycerol into the clay results in an ordered structure with a characteristic diffraction peak at $2 \theta \mathrm{ca} .5^{\circ}$ (Chen \& Evans, 2005). The absence of this diffraction peak from the starch-clay nanocomposites indicates that the glycerol (plasticizer) did not intercalate into the clay layers. Intercalation of glycerol into clay can be prevented by changing the addition sequence of the components (starch, clay, and glycerol). A good dispersion of clays in starch matrix would be achieved by first preparing the nanocomposites in diluted aqueous solutions followed by plasticization.

Fig. 3a and $b$ show the TEM micrographs of starch-clay nanocomposites containing 5\% clay. Exfoliated clay layers coexisting with some clay agglomerates can be seen in the image (Fig. 3a). The clay consists of relatively small tactoids containing less than 5 layers. The clay agglomerates as shown in Fig. 3b are often internally disordered but somewhat aligned. These silicate platelets are distorted, shifted, and skewed relative to one another. The clays show some orientation and this is due to the clay alignment during compression molding. The dispersion of the silicate layers on the micro- and macroscale is equally important. Fig. 3c-f shows the SEM micrographs of the fractured surfaces. A homogeneous surface is observed for both starch and starch nanocomposite indicating that the starch granules were completely disrupted and the clay was dispersed in the polymer matrix (Fig. $3 \mathrm{c}$ and d). No clay aggregation can be seen even at higher magnifications (Fig. 3e and f). Low amount of clay seems to be fairly compatible and miscible with starches resulting in well-dispersed nanocomposites. 

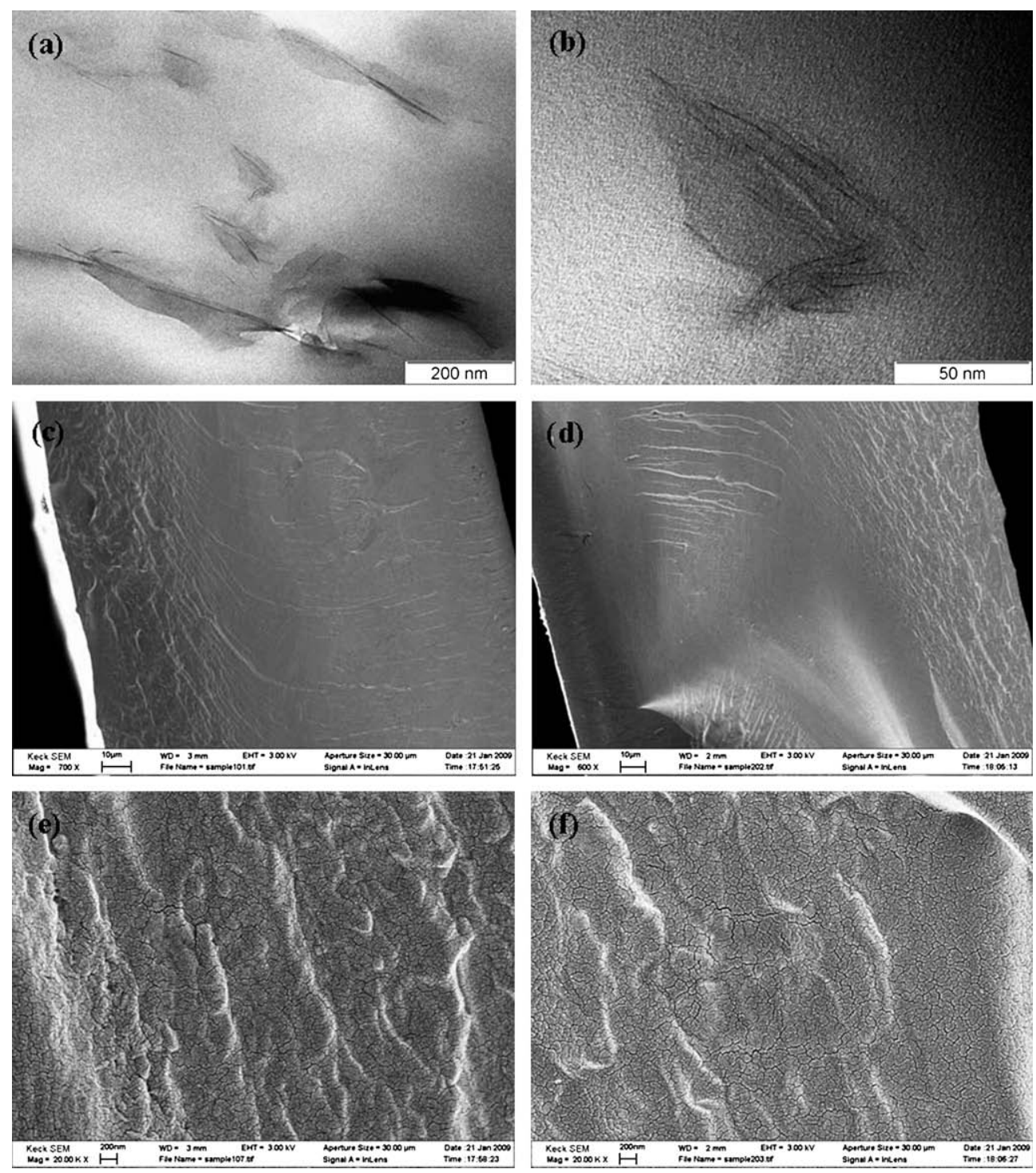

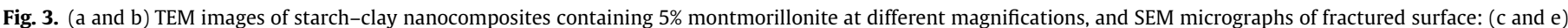
unfilled starch samples and ( $\mathrm{d}$ and f) starch-5\% montmorillonite nanocomposites.

\subsection{Mechanical properties}

The mechanical properties of the starch film and the nanocomposites are shown in Table 1. Both starch-montmorillonite and starch-laponite nanocomposites showed an improvement in Young's modulus and tensile strength compared to the pure matrix. The improvement of Young's modulus for NCS.MMT and NCS.Laponite is $65 \%$ compared to the unfilled starch materials. This is a significant improvement compared to other studies on starch nanocomposites with 5 wt.\% nanoparticles (Chivrac et al., 2008;

Table 1

Mechanical properties of native corn starch and starch-clay nanocomposites containing $5 \%$ clay. The samples were conditioned at $43 \% \mathrm{RH}$ before measurement.

\begin{tabular}{lcll}
\hline Sample & $\begin{array}{l}\text { Young's modulus } \\
(\mathrm{MPa})\end{array}$ & $\begin{array}{l}\text { Tensile strength } \\
(\mathrm{MPa})\end{array}$ & $\begin{array}{l}\text { Elongation at } \\
\text { break }(\%)\end{array}$ \\
\hline NCS & $840 \pm 61$ & $11.82 \pm 0.57$ & $4.62 \pm 1.06$ \\
NCS.MMT & $1390 \pm 166$ & $15.49 \pm 0.67$ & $4.34 \pm 1.15$ \\
NCS.ChitosanMMT & $805 \pm 79$ & $12.46 \pm 1.45$ & $5.35 \pm 1.17$ \\
NCS.Laponite & $1406 \pm 105$ & $15.46 \pm 1.41$ & $3.34 \pm 0.85$ \\
\hline
\end{tabular}

Kampeerapappun et al., 2007; Kvien, Sugiyama, Votrubec, \& Oksman, 2007; Lu, Weng, \& Cao, 2006; Mondragon, Mancilla, \& Rodriguez-Gonzalez, 2008; Pandey and Singh, 2005). The corresponding increase in tensile strength is ca. $30 \%$. The increases in modulus and strength are not accompanied by a decrease in elongation at break. We attribute the significant increases in modulus and strength to the good dispersion of clay in the starch matrix. In contrast, the Young's modulus and tensile strength of starch-chitosanmodified montmorillonite nanocomposites are not significantly different from the neat starch matrix. This difference from the unmodified clays is probably due to the presence of larger agglomerates in the case of chitosan modified clays which behave as larger filler particles rather than independent nanoscale fillers. The larger aggregates reflect the difficulty of dispersing the chitosan exchanged clay in starch due to the stronger interactions between chitosan and clay.

Fig. 4 shows the XRD patterns for starch-clay nanocomposites. All traces show the B-type starch structure with peaks at $2 \theta=17^{\circ}$, $19^{\circ}, 22^{\circ}$, and $24^{\circ}$. The strong diffraction peak of NCS.ChitosanMMT at $2 \theta \sim 20^{\circ}$ is due to both the crystalline structure of chitosan and starch. The A-type crystalline structure of native corn starches was 


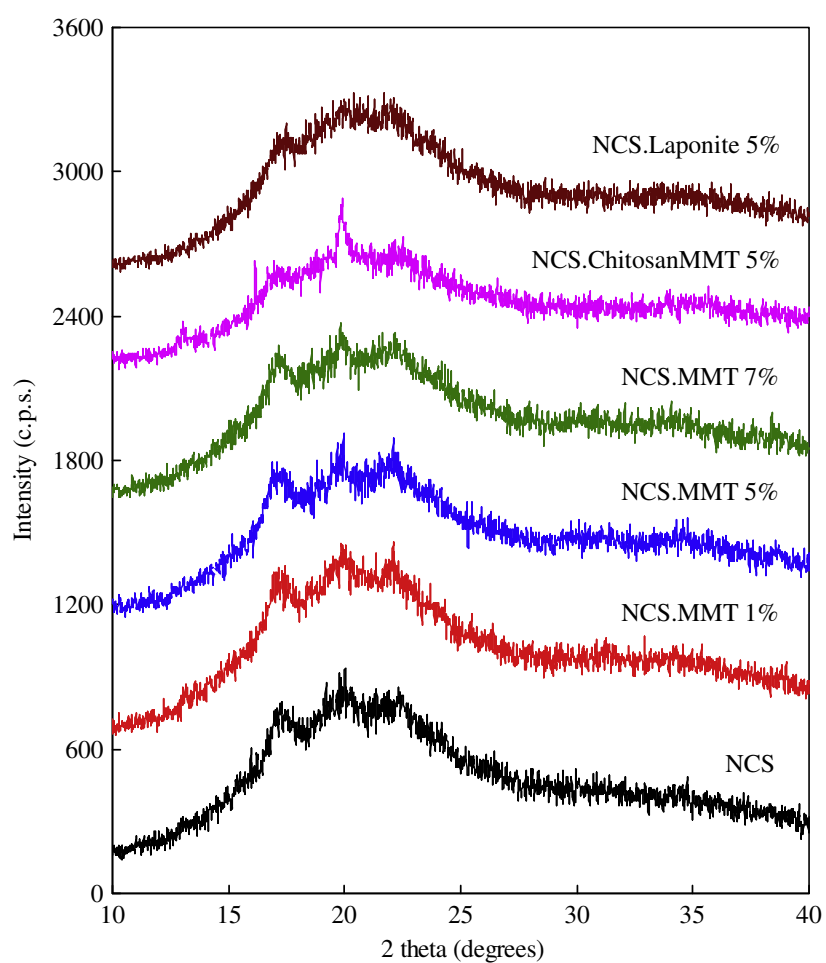

Fig. 4. XRD patterns for starch and various starch-clay nanocomposites containing different amounts of clay.

completely disrupted during the heating process. The starch molecules then recrystallize during compression molding and subsequent storage resulting in the B-type crystalline structure. The addition of clay in the starch matrix didn't significantly influence the type of crystalline structure of starch molecules although the amount of crystallinity (area under the curve) appears somewhat lower in the nanocomposites (see below). Therefore, the crystalline structure of starch appears not to be an important factor determining the mechanical properties of starch-clay nanocomposites.

The incorporation of montmorillonite strongly enhances the stiffness of the starch-clay nanocomposites (Fig. 5). Even at $1 \%$ of clay loading, the increment of Young's modulus is 53\% compared to the unfilled starch matrix. The nanocomposite containing 5\% montmorillonite possesses the highest Young's modulus, showing an increase of $65 \%$. A further increase in montmorillonite content

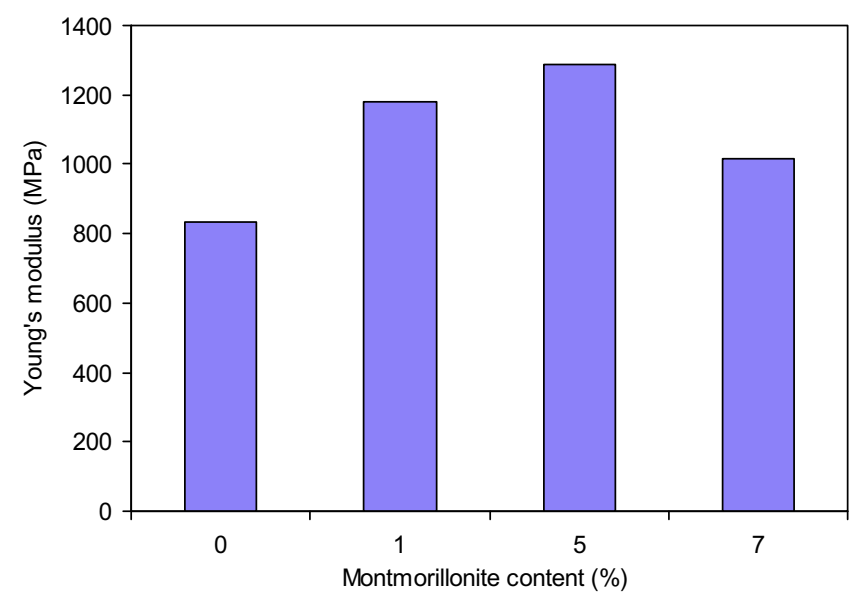

Fig. 5. Young's modulus of starch-montmorillonite nanocomposites containing different clay loading. The samples were conditioned at $43 \%$ RH before measurement. to $7 \%$ brings about a decrease in Young's modulus. We speculate that, as the clay loading increases, the clay dispersion decreases leading to lower degrees of reinforcement. Another possibility is that, as the clay content increases, the crystallinity of the polymer decreases, leading to a lower modulus. Evidence for the effect of clay on the crystallinity of starch is provided in Fig. 4. As can be seen, the crystallinity of starch (area under the curve) seems to be decreasing with increasing clay content. The decrease in crystallinity might more than compensate the reinforcement due to the clay.

Starch-clay nanocomposites with different montmorillonite contents were conditioned at different relative humidity. Changes in the environmental humidity influence the moisture content of starch-clay nanocomposites (Fig. 6). The moisture content is unchanged in the nanocomposites compared to the pure polymer for both clay loadings for $\mathrm{RH}$ up to $75 \%$ (10\% and $25 \%$ for $\mathrm{RH}$ of 43 and 75\%, respectively) (Fig. 6). However, at 97\% RH, the nanocomposites show increased moisture content with clay addition. As expected the modulus of starch decreases with increasing $\mathrm{RH}$ or moisture content (Fig. 7). Water acts as a plasticizer decreasing the glass transition temperature of starch resulting in softer materials. A similar trend is seen for the nanocomposites (Fig. 7). We note that the modulus decrease with increasing $\mathrm{RH}$ is not as steep in the nanocomposites. This behavior suggests that the addition of clay to form nanocomposites might prevent property changes during transportation and storage in starch-based products. Starchclay nanocomposites conditioned at $43 \%$ RH show an improvement in modulus compared to the neat starch matrix. This increase is minimized at $100 \% \mathrm{RH}$ and $0 \% \mathrm{RH}$. While the water uptake is comparable for the neat starch and the nanocomposites, the values diverge at $97 \% \mathrm{RH}$. For example the water content for the nanocomposite containing $5 \%$ clay is $31 \%$ higher than the neat starch. We hypothesize that, at this high water content and because of the hydrophilicity of clay, the water molecules concentrate at the interface resulting in a weaker interface and subsequently lower modulus.

\section{Conclusions}

The main challenges for preparing high performance starchclay nanocomposites include achieving a high level of clay dispersion in the polymeric matrix, finding suitable plasticizers, and controlling the interfacial strength between starch and clay. Well-dispersed starch-clay nanocomposites were prepared by

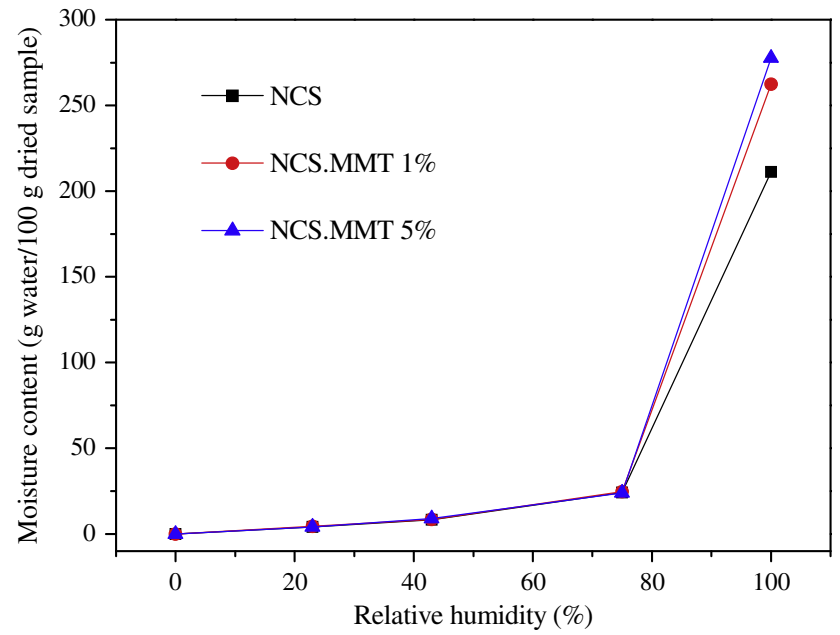

Fig. 6. Moisture adsorption of starch and starch-clay nanocomposites. 


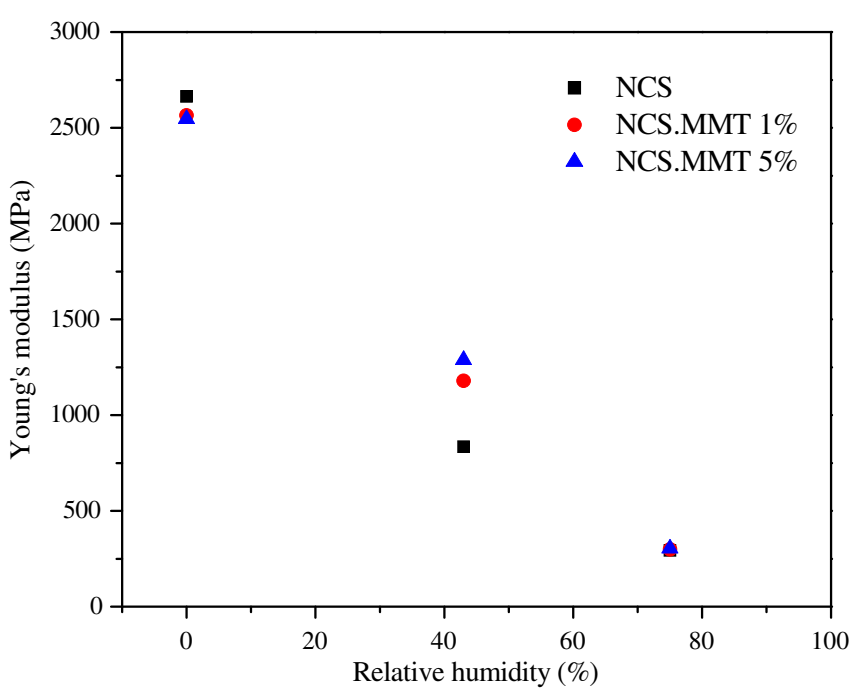

Fig. 7. Effects of relative humidity on Young's modulus of starch-montmorillonite nanocomposites with different clay content.

adding a dilute clay dispersion to a solution of starch followed by coprecipitation in ethanol. In this approach, intercalation of the plasticizer (glycerol) in clay which is required for hot-pressing is minimized. The addition of clay in starch didn't significantly influence the type of crystalline structure of starch molecules, although the amount of crystallinity appears somewhat lower in the nanocomposites. The good dispersion of montmorillonite in the matrix increases the interaction surface area between starch molecules and clays and facilitates stress transfer to the reinforcement phase. This combination leads to improved modulus and strength without a decrease in elongation to break. The increase in modulus and strength is $65 \%$ and $30 \%$, respectively, for the nanocomposite containing 5 wt.\% clay compared to the unfilled starch materials. Further increases in clay result in deterioration in properties most likely due to poorer clay dispersion and lower polymer crystallinity. As the amount of water increases, the modulus of both pure starch and starch nanocomposites decresases, although the change is less pronounced in the nanocomposites suggesting that the addition of clay to form nanocomposites can improve the stability of starch-based products during transportation and storage.

\section{Acknowledgements}

This work was supported by the Grants NSC-96-2313-B-002048-MY2 and NSC-96-2917-I-002-102 from the National Science Council, Taipei, Taiwan. E.P. Giannelis acknowledges the support of Award No. KUS-C1-018-02, made by King Abdullah University of Science and Technology (KAUST). We also thank Chia-Chen (Jason) Fang for the SEM measurements and helpful discussions.

\section{References}

Avella, M., Vlieger, J. J. D., Errico, M. E., Fischer, S., Vacca, P., \& Volpe, M. G. (2005) Biodegradable starch/clay nanocomposite films for food packaging applications. Food Chemistry, 93, 467-474.

Bagdi, K., Muller, P., \& Pukanszky, B. (2006). Thermoplastic starch/layered silicate composites: Structure, intercalation, properties. Composite Interfaces, 13, 1-17.

Chen, B., \& Evans, J. R. G. (2005). Thermoplastic starch-clay nanocomposites and their characteristics. Carbohydrate Polymers, 61, 455-463.

Chiou, B. S., Yee, E., Wood, D., Shey, J., Glenn, G., \& Orts, W. (2006). Effects of processing conditions on nanoclay dispersion in starch-clay nanocomposites. Cereal Chemistry, 83, 300-305.

Chivrac, F., Gueguen, O., Pollet, E., Ahzi, S., Makradi, A., \& Averous, L. (2008) Micromechanical modeling and characterization of the effective properties in starch-based nano-biocomposites. Acta Biomaterialia, 4, 1707-1714.

Chivrac, F., Pollet, E., Schmutz, M., \& Averous, L. (2008). New approach to elaborate exfoliated starch-based nanobiocomposites. Biomacromolecules, 9, 896-900.

Darder, M., Colilla, M., \& Ruiz-Hitzky, E. (2003). Biopolymer-clay nanocomposites based on chitosan intercalated in montmorillonite. Chemistry of Materials, 15, 3774-3780.

Giannelis, E. P. (1996). Polymer layered silicate nanocomposites. Advanced Materials, 8, 29-35.

Huang, M., Yu, J., \& Ma, X. (2006). High mechanical performance MMT-urea and formamide-plasticized thermoplastic cornstarch biodegradable nanocomposites. Carbohydrate Polymers, 63, 393-399.

Kampeerapappun, P., Aht-ong, D., Pentrakoon, D., \& Srikulkit, K. (2007). Preparation of cassava starch/montmorillonite composite film. Carbohydrate Polymers, 67, 155-163.

Kvien, I., Sugiyama, J., Votrubec, M., \& Oksman, K. (2007). Characterization of starch based nanocomposites. Journal of Materials Science, 42, 8163-8171.

Lu, Y. S., Weng, L. H., \& Cao, X. D. (2006). Morphological, thermal and mechanical properties of ramie crystallites-reinforced plasticized starch biocomposites. Carbohydrate Polymers, 63, 198-204.

Ma, X., Yu, J., \& Wang, N. (2007). Production of thermoplastic starch/MMT-sorbitol nanocomposites by dual-melt extrusion processing. Macromolecular Materials and Engineering, 292, 723-728.

Mondragon, M., Mancilla, J. E., \& Rodriguez-Gonzalez, F. J. (2008). Nanocomposites from plasticized high-amylopectin, normal and high-amylose maize starches. Polymer Engineering and Science, 48, 1261-1267.

Pandey, J. K, \& Singh, R. P. (2005). Green nanocomposites from renewable resources: Effect of plasticizer on the structure and material properties of clay-filled starch. Starch/Starke, 57, 8-15.

Park, H. M., Lee, W. K., Park, C. Y., Cho, W. J., \& Ha, C. S. (2003). Environmentally friendly polymer hybrids. Part I. Mechanical, thermal, and barrier properties of thermoplastic starch/clay nanocomposites. Journal of Materials Science, 38 909-915.

Park, H. M., Li, X., Jin, C. Z., Park, C. Y., Cho, W. J., \& Ha, C. S. (2002). Preparation and properties of biodegradable thermoplastic starch/clay hybrids. Macromolecular Materials and Engineering, 287, 553-558.

Paul, D. R., \& Robeson, L. M. (2008). Polymer nanotechnology: Nanocomposites. Polymer, 49, 3187-3204.

Pavlidou, S., \& Papaspyrides, C. D. (2008). A review on polymer-layered silicate nanocomposites. Progress in Polymer Science, 33, 1119-1198.

Perez, C. J, Alvarez, V. A., Mondragon, I., \& Vazquez, A. (2008). Water uptake behavior of layered silicate/starch-polycaprolactone blend nanocomposites. Polymer International, 57, 247-253.

Raquez, J. M., Narayan, R., \& Dubois, P. (2008). Recent advances in reactive extrusion processing of biodegradable polymer-based compositions. Macromolecular Materials and Engineering, 293, 447-470.

Ray, S. S., \& Okamoto, M. (2003). Polymer/layered silicate nanocomposites: A review from preparation to processing. Progress in Polymer Science, 28, 1539-1641.

Wilhelm, H. M., Sierakowski, M. R., Souza, G. P., \& Wypych, F. (2003a). The influence of layered compounds on the properties of starch/layered compound composites. Polymer International, 52, 1035-1044.

Wilhelm, H. M., Sierakowski, M. R., Souza, G. P., \& Wypych, F. (2003b). Starch films reinforced with mineral clay. Carbohydrate Chemistry, 52, 101-110.

Zhao, R., Torley, P., \& Halley, P. J. (2008). Emerging biodegradable materials: Starchand protein-based bio-nanocomposites. Journal of Materials Science, 43, 3058-3071. 